\title{
MODELO DIDÁTICO-PRAXEOLÓGICO PARA ENSINO DE VETORES NO ENSINO MÉDIO: POSSIBILIDADES DE TRABALHOS NA TRANSIÇÃO PARA O ENSINO SUPERIOR
}

\section{DIDACTIC-PRAXEOLOGICAL MODEL FOR TEACHING VECTORS IN HIGH SCHOOL: POSSIBILITIES FOR WORK IN THE TRANSITION TO HIGHER EDUCATION}

\author{
Pedro José Defensor Menezes ${ }^{1}$ \\ Edmo Fernandes Carvalho ${ }^{2}$ \\ Lauriclecio Figueredo Lopes ${ }^{3}$
}

\begin{abstract}
RESUMO: Este artigo traz a proposição de um modelo didático-praxeológico - MPD, para o trabalho com um objeto da Geometria Analítica sob a lente da Teoria Antropológica do Didático - TAD. O objetivo principal foi discutir a possibilidade de trabalho com um modelo didático-praxeológico, embasado na noção de vetores no Ensino Médio, como elemento para mitigar lacunas na transição entre Educação Básica e o Ensino Superior, ou seja, um MDP alternativo aos modelos pautados exclusivamente na Base Nacional Comum Curricular BNCC. O modelo, o objeto e as tarefas propostas são compreendidos pela TAD, que considera como pressuposto a necessidade de tarefas que evoquem a indissociabilidade entre o saber-fazer e o discurso racional que o justifica. Utilizou-se um método inspirado na análise praxeológica para discutir a viabilidade do MDP, a priori. Diante dessas condições, conclui-se que o MDP, pautado na noção de vetores e trabalhado desde o Ensino Médio, pode colocar o estudante em atividade, com praxeologias mais econômicas do ponto de vista cognitivo, nesse nível de escolaridade, mas principalmente no superior.
\end{abstract}

PALAVRAS-CHAVE: Vetores. Ensino de Matemática. Análise Praxeológica.

ABSTRACT: This article proposes a didactic-praxeological model - DPM, for working with an object of Analytical Geometry under the lens of the Anthropological Theory of Didactics - ATD. The main objective was to discuss the possibility of working with a didactic-praxeological model based on the notion of vectors in High School as an element to mitigate gaps in the transition between Basic Education and Higher Education, that is, an alternative DPM to the models based exclusively on the Base Nacional Comum Curricular. The model, the object and the proposed tasks are understood by the TAD, which considers as an assumption the need for tasks that evoke the inseparability between know-how and the rational discourse that justifies it. A method inspired by the praxeological analysis was used to discuss the feasibility of the MDP, a priori. Given these conditions, it is concluded that the MDP based on the notion of vectors worked since high school, can put the student in activity, with praxeologies at this level, but mainly at higher education, which are more economical from a cognitive point of view.

KEYWORDS: Vectors. Teaching of Mathematics. Praxeological Analysis.

\footnotetext{
${ }^{1}$ Escola Estadual El Shadai. E-mail: pedrojdm@gmail.com

(1D) https://orcid.org/0000-0002-3701-8728

2 Universidade Federal do Oeste da Bahia. E-mail: edmo.carvalho@ufob.edu.br

iD http://orcid.org/0000-0002-6959-2652

3 Universidade Federal do Oeste da Bahia. E-mail: lauriclecio@ufob.edu.br

(iD) https://orcid.org/0000-0002-3356-5644

Informações completas da obra no final do artigo
} 


\section{Introdução}

Neste artigo, apresentamos um recorte de uma pesquisa de mestrado, realizada pelo primeiro autor, sob a orientação dos coautores, na qual tomou-se como objetivo propor e analisar um modelo didático-praxeológico para o ensino de objetos da Geometria Analítica - GA, que corroboram a transição entre os níveis básico e superior.

Dessa maneira, consideraram-se como problemática didática os aspectos implícitos nas práticas institucionais (escolares ou aquelas relativas à formação docente) no que se refere à transição do Ensino Médio para o Superior. Segundo Menezes (2021), as praxeologias dos estudantes em curso de licenciatura em Matemática, por vezes, são construídas sem um alicerce sólido daquelas possivelmente estudadas na Educação Básica, como no caso do estudo de vetores na Geometria Analítica. Mas isso ocorre, também, em relação a objetos estudados, como no caso das equações das retas, por exemplo.

Tal fato nos chamou a atenção para o que ocorre na transição de um nível de ensino para o outro. O que, por sua vez, levou nosso olhar para os documentos que registram as praxeologias dos dois níveis. Desse modo, a utilização do livro didático nessa investigação se justificou devido à oportunidade de colocar lado a lado as praxeologias que se constroem no Ensino Médio e Superior. Entretanto, neste recorte da investigação, discutido nesse artigo, não tratamos das análises dos livros didáticos do Ensino Médio e Superior, mas optou-se por apresentar as tarefas construídas e/ou adaptadas por Menezes (2021).

É verdade que estamos falando de duas modalidades de ensino diferentes, por isso, a partir daqui, chamaremos de instituições, com objetivos distintos, nos quais naturalmente não encontramos uma abordagem exatamente igual dos saberes da GA. No entanto, há que se considerar que devem existir pontos em comum entre os objetos estudados em ambas as instituições, sendo o Ensino Superior um local para aprofundamento desse estudo.

No entanto, nesse manuscrito, nos propomos a responder quais elementos um modelo didático deve considerar para mitigar lacunas da transição entre Ensino Médio e Superior no estudo de objetos da GA? Isso implica em apresentar um esboço de um modelo didático de referência para ensino de um objeto específico como forma de aprofundar a discussão, o que é feito por meio da apresentação de situações em que o estudo de vetores 


\section{ENSIN@UFMS 2021}

ISSN 2525-7056

amplificaria as técnicas de resolução das tarefas de tal modelo, além de se constituir um instrumento de economia cognitiva e fator relevante para mitigar os problemas da transição interníveis.

Para alcançar o objetivo proposto neste artigo, os argumentos apresentados se alicerçam na Teoria Antropológica do Didático - TAD (CHEVALLARD, 1999), que também se constitui como referencial metodológico, haja vista o método de análise (praxeológica) adotado surgir no seio dessa teoria.

Outrossim, a partir de uma análise inspirada na praxeológica a priori, mostraremos, neste artigo, que um modelo didático para o Ensino Médio pautado na apresentação de vetores pode suavizar a transição do ensino básico para o superior, ou seja, o modelo de ensino de GA do nível médio não estaria somente alicerçado no plano $R^{2}$, mas também no espaço.

\section{Referenciais Teóricos e abordagem metodológica do estudo}

Faz-se necessária, para compreensão da investigação cujo recorte é apresentado nesse artigo, a discussão de alguns conceitos que são utilizados e que impactaram na produção dos resultados da pesquisa. Um desses conceitos é o de modelo didático, o qual nós consideramos a primeira noção de base da TAD, fundante para este trabalho.

Assim, compreendemos um modelo didático como uma tentativa de representar os fazeres pedagógicos dos professores (PORLÁN et al, 1997; GARCÍA PÉREZ, 2000). Além disso, a ideia de modelo didático também está presente quando nos referimos às tomadas de decisões dos professores, que nem sempre são conscientes, mas que, certamente, integram o fazer pedagógico destes, carregados de crenças, valores e conhecimentos didáticos (GIL-PÉREZ; CARVALHO, 1995).

$\mathrm{Na}$ Epistemologia experimental, mais conhecida como Didática da Matemática, fazemos uso do termo modelo didático, mas sem uma definição explícita do que este termo significa. No entanto, encontramos esboços de uma definição em Lucas et al. (2014). Assim, na investigação em tela, entendemos um modelo didático como um esquema mediador entre o pensamento e a realidade social na qual o professor está inserido, estrutura na qual se organiza os conhecimentos docentes em torno da missão de difundir os saberes escolares, embasados na epistemologia geral da ciência a ser ensinada. 
Vale ressaltar que um modelo didático às vezes assume um status de modelo dominante e termina sendo aquele adotado por muitos docentes, independentemente da sua realidade social. De qualquer forma, segundo Chrobak (2006), um modelo didático tem caráter provisional e não representa propriamente a realidade social do professor na tarefa de difundir os saberes escolares, mas uma aproximação com uma determinada realidade.

Do mesmo modo, a noção de modelo didático, de forma simples, nos permite abordar a complexidade da realidade escolar, ao passo que nos auxilia na proposição de estratégias para intervir em tal realidade (GARCÍA PÉREZ, 2000). É possível, na literatura, encontrarmos referência ao modelo didático do professor no sentido de concepções epistemológicas do professor, como em Porlán et al. (1997).

No entanto, neste artigo, vamos nos ater a ideia mais ampla de modelo didático (modelo dominante) que revela praxeologias didáticas e matemáticas, não somente de um professor, mas aquilo que foi culturalmente construído e é usado nos fazeres escolares. De maneira mais específica, nos referimos a modelo didático-praxeológico para chamar atenção de que, em uma aula de matemática, o didático não se dissocia do matemático (praxeologia matemática) e é sobre tal modelo que manteremos o foco.

A segunda noção fundante, para compreensão da proposta aqui apresentada, é a de praxeologia. Chevallard (1999), autor da Teoria Antropológica do Didático (TAD), apresenta a referida noção como uma maneira de modelar a atividade matemática de uma pessoa em um determinada instituição. Trata-se do estudo de ações humanas em instituições, mas especificamente da atividade de pessoas diante da matemática modelada em dois blocos: saber-fazer e o saber. Esses blocos deveriam, na teoria, ser indissociáveis. Entretanto, na prática, isso não é tão comum.

$\mathrm{Na}$ atividade matemática, uma atividade humana como outra qualquer, uma pessoa é colocada frente a uma tarefa para qual apresentará técnica(s); isso constitui o bloco saber-fazer. $\mathrm{Na}$ apresentação de técnica(s), espera-se que a pessoa tente acessar um bloco mais abstrato, o do logos ou tecnológico-teórico que, na prática, se constitui como discurso racional que justifica a(s) técnica(s). Essas noções se justificam na discussão realizada na próxima seção, na medida em que, aponta-se o objeto matemático vetor como ingrediente da técnica, mas também como discurso que justifique esta.

A terceira noção fundamental, empregada na discussão sobre modelos didáticos, é a de relação institucional e pessoal. Um saber, vetores, por exemplo, é um objeto do saber 
de uma instituição a princípio, se for reconhecido por ao menos uma pessoa desta. Podemos falar de algumas relações institucionais que envolvem pessoa, saber e a própria instituição, mas focaremos na relação pessoal que pode ser alcançada por meio de uma possível implementação do modelo didático aqui proposto.

A ideia do modelo, apresentado a seguir, considera que a relação pessoal dos estudantes no ensino básico e superior com os saberes da Geometria Analítica (GA) precisa ser um ponto crucial da discussão sobre o processo de transição entre os níveis de ensino. Uma vez identificadas algumas fragilidades dessa relação, as tarefas que componham o modelo didático, também praxeológico, podem ser direcionadas; é o que se pretende com a análise feita em torno da ideia de ensino de vetores no Ensino Médio e do tipo de tarefa proposta e analisada neste artigo.

Destarte, a hipótese da parte da investigação que compõe esse recorte sugere que o modelo didático-praxeológico alternativo, apresentado para o ensino de objetos da GA, tem condições de promover alterações significativas nas relações pessoais de estudantes no Ensino Médio e mitiga as dificuldades na transição para o Ensino Superior, promovendo maior aproximação com conceitos a partir da iniciativa de resolver e propor problemas, conjecturar e questionar a natureza do conhecimento estudado; isto porque o modelo em jogo talvez possa estimular tais habilidades pelo tipo de tarefa proposta.

No que tange os aspectos metodológicos deste estudo, pautado em Garnica (2004), destacam-se a abordagem que é de natureza qualitativa; e, como instrumento, a proposição de modelo didático-praxeológico, cuja análise aproxima-se da praxeológica a priori. $\mathrm{O}$ modelo é constituído basicamente de tarefas cujas técnicas de resolução estão embasadas numa visão vetorial. Tais tarefas foram elaboradas levando-se em consideração as possíveis conexões que podem se estabelecer no Ensino Médio.

Quanto à aproximação com a análise praxeológica, proposta por Matheron (2000), destacam-se as tarefas, as técnicas e, na sequência, o discurso racional que justifica as mesmas, apontando-se as razões para sua integração ao Ensino Básico e as possíveis consequências didáticas.

A Figura 1, a seguir, representa os passos da investigação de modo geral, ou seja, um desenho da investigação que começa no levantamento de questões docentes, normalmente pautadas na observação direta de alguns fatos da sala de aula, e, no caso da análise inspirada na modelo praxeológico, consiste em estudar praxeologias matemáticas 
que podem ser construídas a partir da experimentação de tarefas do MPD proposto; em outras palavras, uma análise do tipo a priori. O termo é emprestado de uma etapa da Engenharia Didática, embasada em Artigue (1988), mas não foi adotada tal abordagem metodológica.

Figura 1. Desenho da pesquisa

\section{Abordagem qualitativa - Desenho da pesquisa}

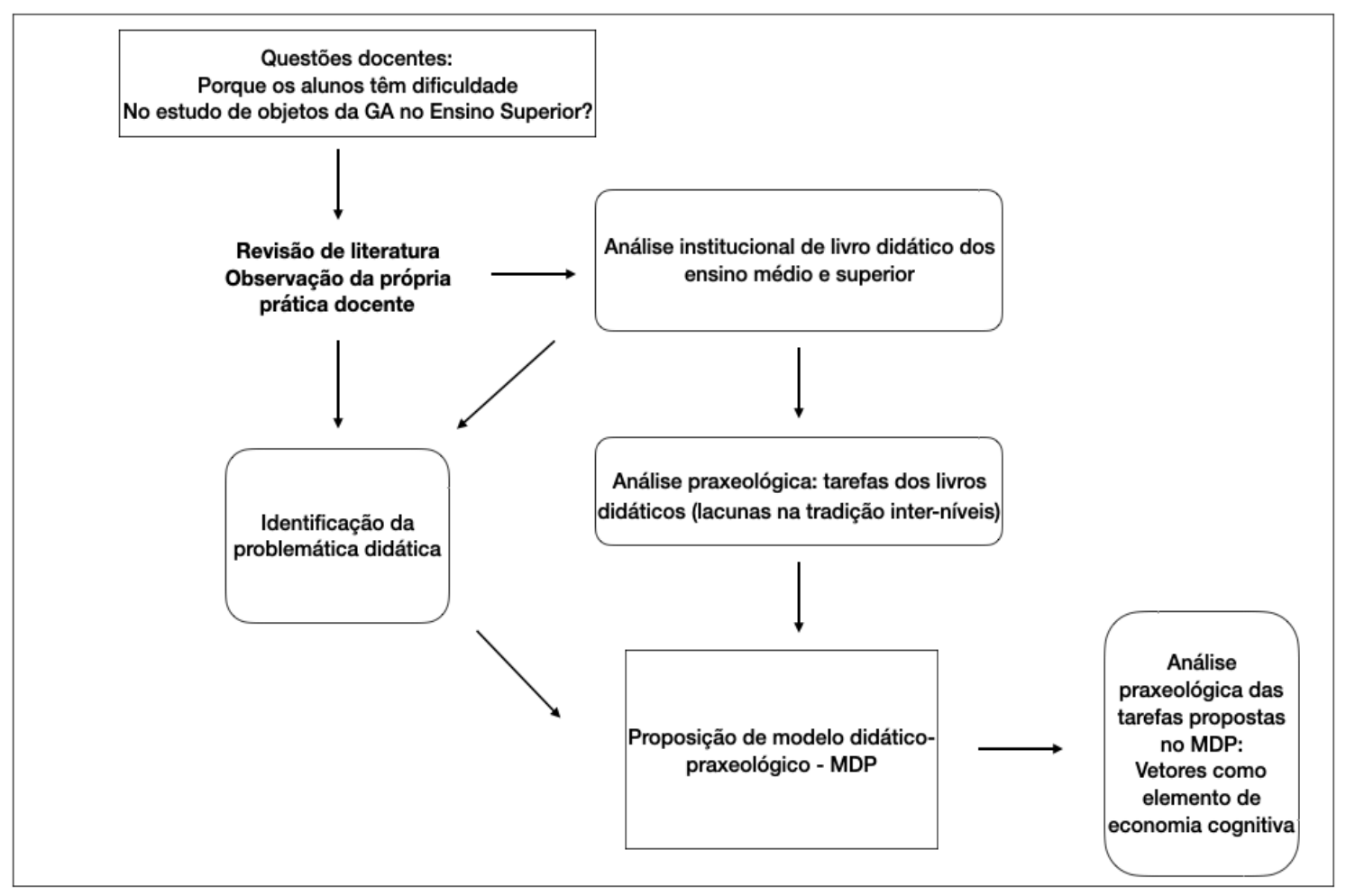

Fonte: Os autores.

Cabe ressaltar que a revisão de literatura (não sistemática) contribuiu para melhor delinear o objeto de investigação e configurou a identificação da problemática didática. De acordo com Henriques, Attie e Farias (2007), contribuiu, ainda, para a compreensão do instrumento a análise institucional, utilizada de diferentes maneiras nas pesquisas no âmbito da Didática da Matemática. As tarefas dos livros passaram por um processo de análise (inspirado na análise praxeológica) e isso foi fundamental para a elaboração/adaptação de tarefas que compõem o MDP que está embasado na visão vetorial, significando uma mudança no Ensino Médio do plano $\left(\mathrm{R}^{2}\right)$ para o plano e espacial 
$\left(R^{2}\right.$ e $\left.R^{3}\right)$, o que se acredita ser uma importante ferramenta de agilidade de raciocínio da resolução das tarefas, definida por Grendene e Melo (2008) como economia cognitiva, o que pode ser visto na seção seguinte.

\section{Apresentação do MDP e análise de suas tarefas}

No centro do MDP, está a noção de vetores. Dessa maneira, primeiro apresentamos uma explanação básica do ponto de vista matemático sobre a parte de vetores que julgamos apropriados ao Ensino Médio e como esta noção poderia se relacionar com os demais conceitos, já tradicionalmente abordados no ensino básico. Também discutimos as vantagens e desvantagens do uso de vetores na resolução de algumas situações (tarefas), mostrando ser viável a abordagem desse saber nessa instituição (EM), o que pode facilitar a atividade matemática do estudante num curso de GA no Ensino Superior, quando estiver trabalhando no plano $\left(R^{2}\right)$ e no espaço $\left(R^{3}\right)$.

Parafraseando Steinbruch (1987), entende-se vetor (no plano) como sendo uma classe de segmentos de reta orientados de modo que dentro da mesma classe todos os segmentos possuem o mesmo comprimento, mesma direção e mesmo sentido. $O$ comprimento é o tamanho do segmento, usualmente chamado de módulo do vetor; a direção é dada pela inclinação do segmento com relação à horizontal, e o sentido está relacionado à orientação do segmento. Dentro de uma mesma classe, qualquer segmento orientado representa um único vetor. Geometricamente, um vetor é representado por um segmento de reta com uma seta em uma das extremidades desenhadas no plano cartesiano. Esta seta indica o sentido do vetor. Na Figura 2, a seguir, ilustramos alguns vetores e, em seguida, tercemos alguns comentários importantes. 
Figura 2. Vetores no Plano

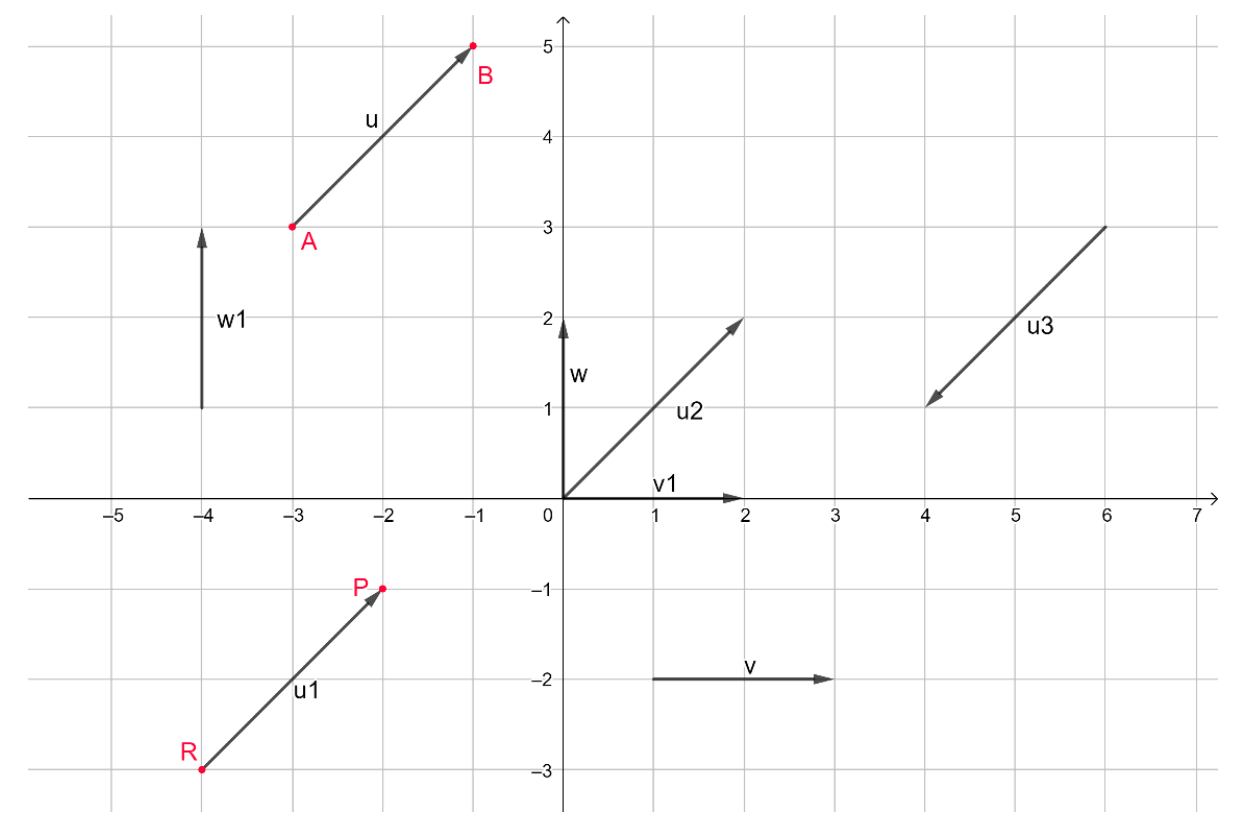

Fonte: Os autores com uso do Geogebra.

Observe que os vetores $u, u 1, u 2$ e $u 3$ possuem o mesmo tamanho (mesmo módulo), a mesma inclinação com relação ao eixo $x$ (mesma direção), mas o vetor $u 3$ possui sentido diferente dos demais. Neste caso, afirmamos que os vetores $u, u 1$ e $u 2$ são iguais. Já o vetor $u 3$ possui sentido oposto aos demais vetores. Nessa mesma ideia, os vetores $v$ e $v 1$ são iguais bem como $w$ e $w 1$. Geometricamente, o vetor não possui posição fixa no plano cartesiano. Porém, algumas vezes, os vetores podem ser determinados por pontos, um ponto de partida e um ponto de chegada. $O$ vetor $u 1$ poderia ser denotado por $R P$, no qual deixaria claro o ponto de inicio e o ponto final do vetor. Reforçamos, porém, que $u=u 1=u 2=R P=A B$.

Algebricamente, um vetor $u$ no plano é descrito por suas coordenadas, da seguinte forma, por $u=(a, b)$. O valor de $a$ é diferença da coordenada $x$ entre as extremidades final e inicial do vetor e $b$ é a diferença das coordenadas $y$ entre extremidade final e inicial do vetor. Se $u=A B$, então $u=(a, b)=\left(x_{B}-x_{A}, y_{B}-y_{A}\right)$. Na Figura 2, temos $u=$ 
$(2,2), v=(2,0), w=(0,2)$ e $u 3=(-2,-2)$. Um caso particular de vetor ocorre quando as extremidades do vetor coincidem; neste caso, o vetor é representado por um ponto e suas coordenadas são iguais a $(0,0)$. Este vetor é chamado de vetor nulo.

Uma vez entendido o conceito de vetor, apresentaremos a definição de três operações envolvendo vetores e citaremos alguns resultados básicos que utilizaremos nos exemplos apresentados a seguir.

Sejam $u=(a, b)$ e $v=(c, d)$ dois vetores e $k$ um número real (chamado escalar), definimos:

a) Adição de vetores: $u+v=(a+c, b+d)$;

b) Multiplicação por escalar: $k u=(k a, k b)$;

c) Produto Interno ou Produto escalar: $u \cdot v=a c+b d$.

Da teoria vetorial citamos duas propriedades básicas: sejam $u=(a, b)$ e $v=(c, d)$ dois vetores não nulos.

i. Os vetores $u$ e $v$ são paralelos (possuem a mesma direção) se, e somente se, um deles é múltiplo do outro, ou seja, existe $k \in R, k \neq 0$ tal que $u=k v$.

ii. Os vetores $u$ e $v$ são perpendiculares (formam um ângulo de 90 graus quando desenhados com mesma extremidade inicial) se, e somente se, $u \cdot v=0$.

Também é possível deduzir a equação geral e reduzida da reta com uso de vetores. Sejam $r$ uma reta, $v=(a, b)$ um vetor não nulo e paralelo a reta e $P\left(x_{0}, y_{0}\right)$ um ponto qualquer de $r$.

Figura 3. Posições entre vetores

Fonte: Os autores com uso do Geogebra. 
Seja $Q(x, y)$ um ponto arbitrário de $r$. Temos que os vetores $v$ e $P Q$ são paralelos, neste caso, existe $t$ real tal que $P Q=t v$. Isso nos diz que vale $\left(x-x_{0}, y-y_{0}\right)=$ ( $t a, t b)$, ou seja, $x-x_{0}=a t$ e $y-y_{0}=b t$. Podemos isolar $t$ nas duas equações $\mathrm{e}$ igualar, desde que a e b sejam não nulos. Teremos assim, $\frac{x-x_{0}}{a}=\frac{y-y_{0}}{b}$, e assim, $b\left(x-x_{0}\right)=a\left(y-y_{0}\right)$ e donde obtemos $b x-a y+a y_{0}-b x_{0}=0$. Fazendo $A=b$, $B=-a$ e $C=a y_{0}-b x_{0}$ temos $\boldsymbol{A} \boldsymbol{x}+\boldsymbol{B} \boldsymbol{y}+\boldsymbol{C}=0$ que é a equação geral da reta $r$. Isolando $y$ nessa equação temos $\boldsymbol{y}=\boldsymbol{m} \boldsymbol{x}+\boldsymbol{n}$, que é a equação reduzida da reta, em que $m=-\frac{A}{B}=\frac{b}{a}$ e $n=-\frac{C}{A}$.

Sobre estas equações da reta fazemos dois comentários:

(i) o vetor $w=(A, B)$ é perpendicular a reta, pois $w \cdot v=(A, B) \cdot(a, b)=(-b, a) \cdot(a, b)=$ $-a b+a b=0$

(ii) o vetor $u=(1, m)$ é paralelo a reta, pois $v=(a, b)=a(1, b / a)=a(1, m)=k u$, com $k=a$. Veja que do comentário (i) concluímos que dado um vetor $v=(a, b)$, o vetor $v_{1}=$ $(-b, a)$ é perpendicular a $v$.

A partir da igualdade $\left(x-x_{0}, y-y_{0}\right)=(t a, t b)$, é possível definir as equações paramétricas da reta, o que ampliaria o leque de tarefas e aplicações. Porém, neste artigo, não abordaremos este tópico.

Com a integração de vetor no plano na Matemática do Ensino Médio, já seria possível responder várias tarefas de Geometria Analítica tanto com o uso de vetores quanto sem, ampliando, assim, o leque de opções de resolução das situações para os estudantes. Vejamos alguns exemplos de tarefas com comentários das consequências didáticas de sua experimentação após a resolução.

Tarefa 1: verificar se os pontos $A=(2,1), B=(14,10)$ e $C=(18,13)$, dispostos na figura 4 , são colineares. 
Figura 4. Três pontos colineares T1

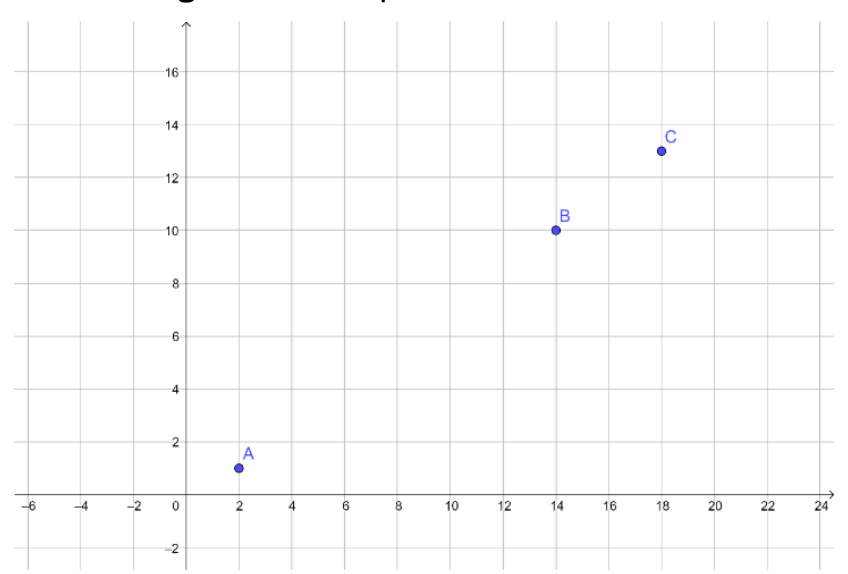

Fonte: Os autores com uso do Geogebra.

Solução. Sabemos que três pontos são colineares quando estes dois a dois determinam uma mesma reta.

Resolução 1 - técnica 1 (sem vetores): neste caso, se pontos forem colineares, dois a dois definem um segmento contido na reta; por exemplo, os segmentos $A B$ e $B C$ estão contidos na reta e sua inclinação deve ser a mesma da reta, isto é, o coeficiente angular de $A B$ e de $B C$ deve ser o mesmo. Se isso ocorrer, os três pontos são colineares. Coeficiente angular do segmento $A B$, que denotaremos por $m_{A B}$

$$
m_{A B}=\frac{y_{B}-y_{A}}{x_{B}-x_{A}}=\frac{10-1}{14-2}=\frac{9}{12}=\frac{3}{4}
$$

Coeficiente angular do segmento $B C$, que denotaremos por $m_{B C}$

$$
m_{B C}=\frac{y_{C}-y_{B}}{x_{C}-x_{B}}=\frac{13-10}{18-14}=\frac{3}{4}
$$

Como há igualdade entre os coeficientes, concluímos que os três pontos são colineares.

Nessa resolução, está implícita a ideia de que a reta determinada por $A$ e $B$ e a reta determinada por $B$ e $C$ possuem a mesma inclinação, ou seja, são paralelas. Como elas possuem o ponto $B$ em comum, elas devem ser iguais e, assim, conclui-se que $A, B$ e $C$ definem uma mesma reta e, portanto, são pontos colineares. A Figura 5, a seguir, ilustra essa resolução. 
Figura 5. Resolução geométrica tarefa 1

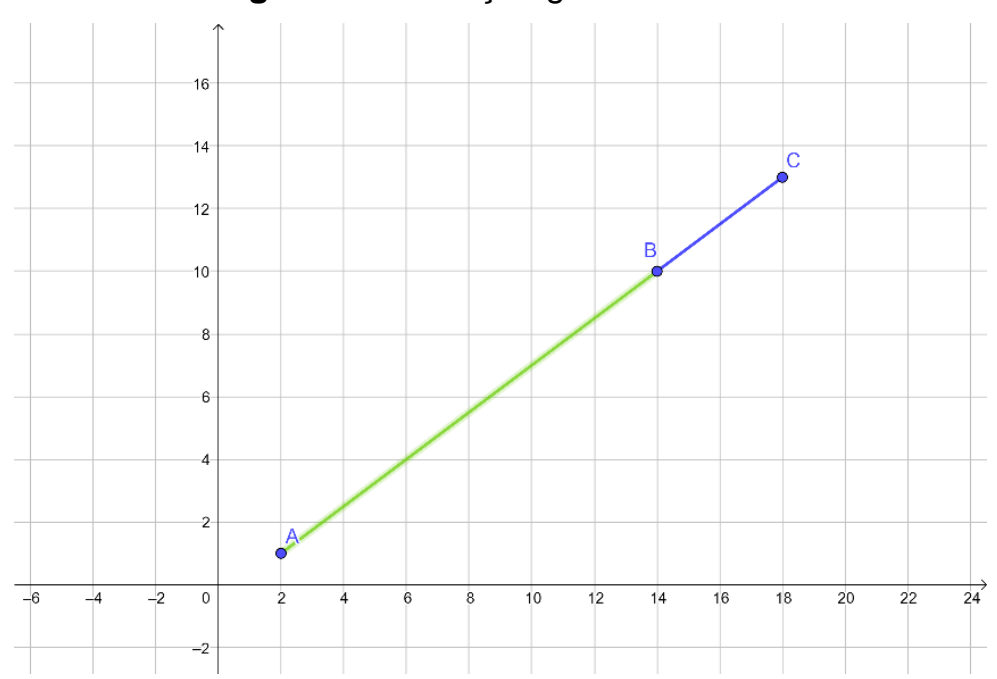

Fonte: Os autores com uso do Geogebra.

Resolução 2 - técnica 2 (sem vetores): uma alternativa na resolução desta questão, que é trabalhada no Ensino Médio, é verificar se o determinante abaixo é igual a zero. Se for, os pontos são colineares, se não for, os pontos não o são.

$$
\operatorname{det}\left(\begin{array}{lll}
x_{A} & y_{A} & 1 \\
x_{B} & y_{B} & 1 \\
x_{C} & y_{C} & 1
\end{array}\right)=\operatorname{det}\left(\begin{array}{ccc}
2 & 1 & 1 \\
14 & 10 & 1 \\
18 & 13 & 1
\end{array}\right)=0 .
$$

Isso nos diz que os pontos são colineares. Essa resolução não deixa claro para o estudante os motivos que, de fato, justificam que se o determinante é igual a zero, isso implica que os pontos são colineares. O que a torna uma resolução amparada no processo de memorização de fórmula.

Resolução 3 - técnica 3 (com uso de vetores): primeiro, é preciso dizer que tal técnica pode ser considerada não padrão para o ensino básico, haja vista o fato de vetor não ser um objeto com lugar e função definidos nesse nível de ensino. Ademais, da Figura 6, que ilustra a representação geométrica dos pontos, podemos considerar os vetores $A B$ e $A C$. Se esses vetores forem paralelos, as retas definidas por $A$ e $B$ e por $A$ e $C$ seriam paralelas com o ponto $A$ em comum; logo, seria a mesma reta e daí $A, B$ e $C$ seriam pontos colineares. Temos $A B=(12,9)$ e $A C=(16,12)$. Estes vetores serão paralelos se existir $k$ real não 
nulo tal que $A C=k A B$, ou seja, $(16,12)=k(12,9)$, isto nos dá, $(16,12)=(12 k, 9 k)$, de onde obtemos $12 k=16$ e $9 k=12$. Obtemos que $k=4 / 3$ satisfaz as duas igualdades. Portanto, $A B$ e $A C$ são vetores paralelos, implicando, assim, que $A, B$ e $C$ são colineares.

Figura 6. Ilustração de solução da tarefa 1

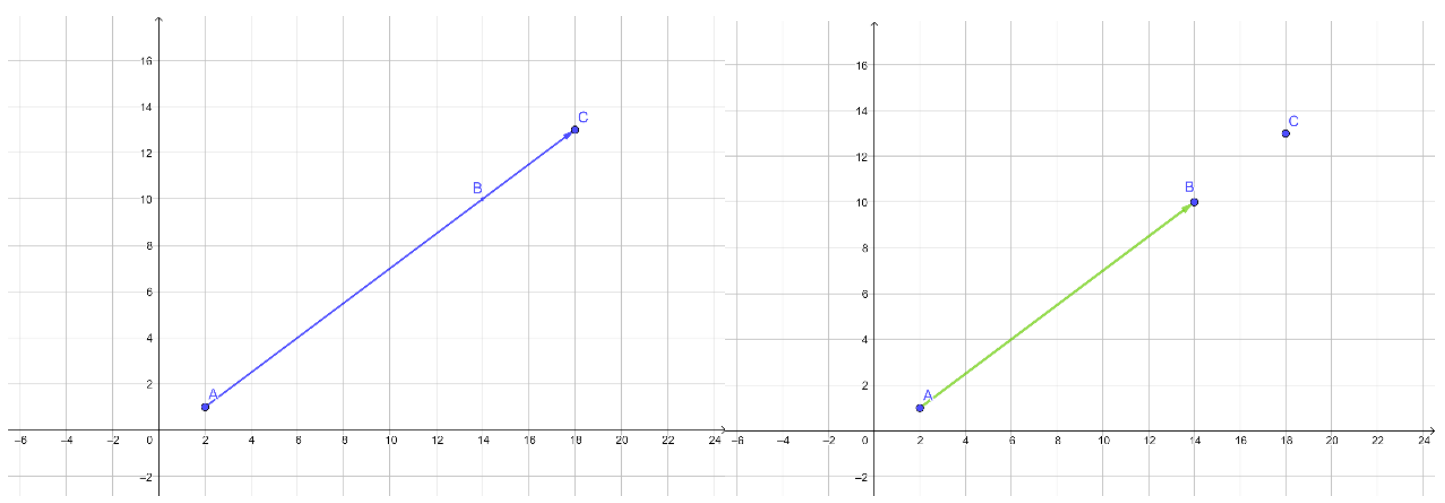

Fonte: Os autores com uso do Geogebra.

Nessa tarefa, consideramos que a resolução 1 e a resolução 3 são as duas resoluções que trazem um significado geométrico para as praxeologias do estudante, fazendo com que seja mais compreensível e até mesmo reforce conhecimentos prévios já estudados, como, por exemplo, o conceito de paralelismo. Em termos de grau de dificuldade, apontamos que ambas as resoluções se equivalem, visto que em uma é necessário conhecer a fórmula do coeficiente angular e a outra necessitaria lembrar-se do resultado $i$, citado anteriormente. Claramente, a inclusão dos vetores não dificultaria a resolução da questão. Como observação complementar, comentamos que na resolução 3 poderiam ter sido utilizados os vetores $A B$ e $B C$, de modo a se ter uma compatibilidade maior com a resolução 1; porém usamos os vetores $A B$ e $A C$ para ilustrar que nas resoluções 1 e 3 qualquer combinação entre os segmentos $A B, B C$ e $A C$ para o cálculo dos coeficientes angulares e qualquer combinação entre os vetores $A B, A C$ e $B C$, respectivamente, seria válida, dando, assim, maior liberdade de escolha ao estudante. 
Tarefa 2: verificar se os pontos $\mathrm{P}(3,1), \mathrm{Q}(5,3)$ e $\mathrm{R}(2,6)$ são vértices de um triângulo retângulo.

Solução. Após a marcação dos pontos no plano cartesiano, obteríamos a Figura 7, apresentada a seguir, pela qual deduziríamos que se o triângulo é do tipo retângulo, o ângulo reto deve ser o ângulo com vértice em $\mathrm{Q}$.

Figura 7. llustração da solução da tarefa 2

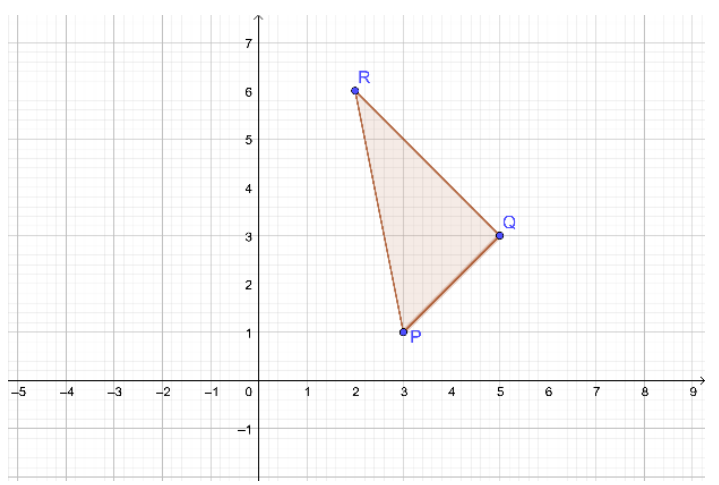

Fonte: Os autores com uso do Geogebra.

Resolução 1 - técnica 1 (sem vetores). Para verificar se o ângulo interno do vértice $Q$ mede $90^{\circ}$, verificaremos se os coeficientes dos segmentos $Q P$ e $Q R$, respectivamente denotadas por $m_{Q P}$ e $m_{Q R}$, satisfazem $m_{Q P} \cdot m_{Q R}=-1$. Se isso for satisfeito, os segmentos são perpendiculares. Temos que:

$$
m_{Q P}=\frac{y_{Q}-y_{P}}{x_{Q}-x_{P}}=\frac{3-1}{5-3}=1 \text { e } m_{Q R}=\frac{y_{Q}-y_{R}}{x_{Q}-x_{R}}=\frac{3-6}{5-2}=\frac{-3}{3}=-1 .
$$

Claramente vale $m_{Q P} \cdot m_{Q R}=-1$ e assim o triângulo é retângulo em $Q$.

Resolução 2 - técnica 2 (com uso de vetores). Para verificar se o triângulo é triângulo retângulo em $Q$, verificaremos se os vetores $Q P$ e $Q R$ são perpendiculares, ou seja, se $\overrightarrow{Q P} \cdot \overrightarrow{Q R}=0$. Temos que $\overrightarrow{Q P}=(-2,-2)$ e $\overrightarrow{Q R}=(-3,3)$, e $\overrightarrow{Q P} \cdot \overrightarrow{Q R}=-2 \cdot(-3)+$ $(-2) \cdot(3)=0$. Portanto, temos um triângulo retângulo em $Q$.

Nessa tarefa, ambas as resoluções são econômicas do ponto de vista cognitivo, ou seja, não apresentariam grandes dificuldades para seu uso, exceto o fato de uma dada técnica não ter função na instituição Ensino Médio. 
Tarefa 3: determinar a equação da reta r que contém os pontos $P(2,3)$ e $Q(1,5)$. A Figura 8 , a seguir, ilustra a referida tarefa.

Figura 8. llustração da tarefa 3

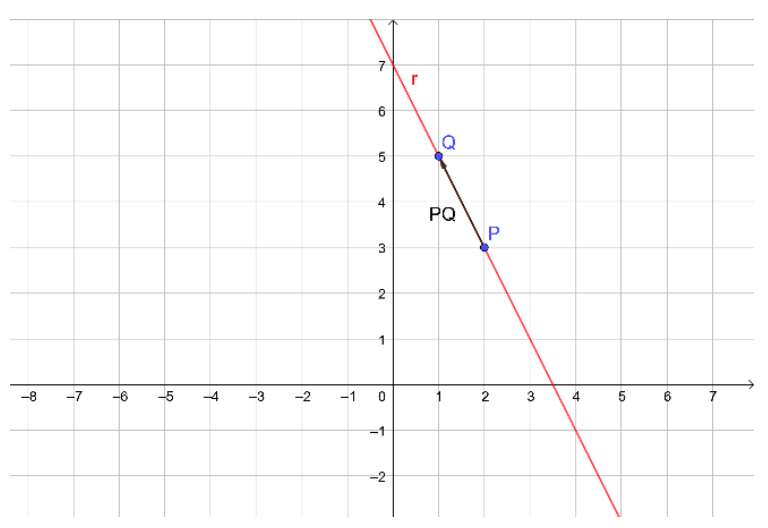

Fonte: Os autores com uso do Geogebra.

Resolução 1 - técnica 1 (sem vetores). Para este problema, existe a famosa fórmula $y-y_{0}=\frac{y_{1}-y_{0}}{x_{1}-x_{0}}\left(x-x_{0}\right)$ para a equação da reta $r$. Neste caso, basta escolher qual ponto entre $P$ e $Q$ teria coordenadas $\left(x_{0}, y_{0}\right)$ e qual teria $\left(x_{1}, y_{1}\right)$. Apos a escolha, basta substituir na fórmula e obteremos a equação da reta. Se escolhermos $\left(x_{0}, y_{0}\right)=P(2,3)$ teremos $\left(x_{1}, y_{1}\right)=Q(1,5)$. Assim, $y-3=\frac{5-3}{1-2}(x-2)$ o que nos dar $y-3=-2 x+$ 4 , logo $2 x+y-7=0$ é a equação geral e $y=-2 x+7$ é a equação reduzida.

Resolução 2 - técnica 2 (com uso de vetores). Pelo fato da reta conter os pontos $P$ e $Q$, temos que ela é paralela ao vetor $P Q=(1-2,5-3)=(-1,2)$ e é perpendicular ao vetor $w=(A, B)=(-2,-1)$. Substituindo na equação $A x+B y+C=0$, temos $-2 x-y+C=0$. Para determinar o valor de $C$, basta substituir as coordenadas do ponto $P$ ou do ponto $Q$, pois ambos estão na reta e, obrigatoriamente, suas coordenadas devem satisfazer a equação da reta. Escolhendo $P$, temos $-2(2)-(3)+C=0, \operatorname{logo}, C=7$. Assim, $-2 x-y+7=0$ ou $2 x+y-7=0$ são as equações gerais da reta. Isolando $y$, temos que $y=-2 x+7$ é a equação reduzida. 
Tarefa 4: considere os $P(2,3)$ e $Q(1,5)$. Determine a equação da reta $S$ que intercepta a origem do plano cartesiano e é perpendicular a reta que contém $P$ e $Q$.

Solução. Considerar a Figura 9, a seguir, que ilustra a tarefa.

Figura 9. ilustração para solução da tarefa 4

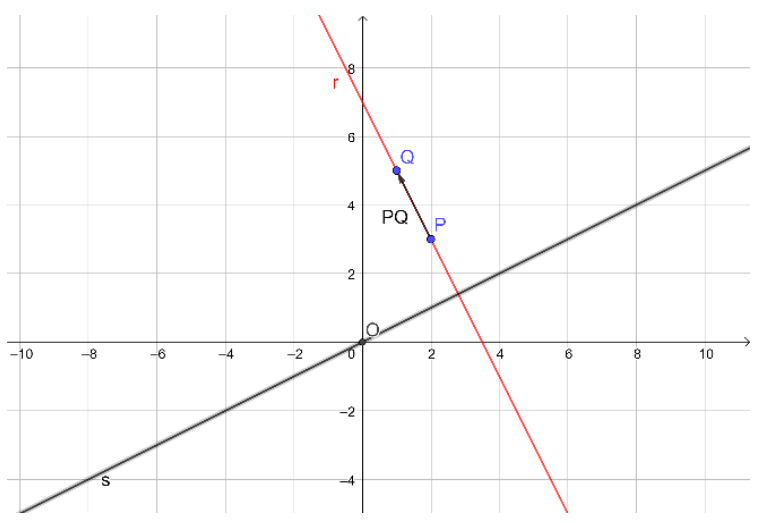

Fonte: Os autores com uso do Geogebra.

Resolução 1 - técnica 1 (sem vetores). Nessa tarefa, seria necessário determinar o coeficiente angular $m_{r}$ da reta $r$ que contém os pontos $P$ e $Q$ por meio da fórmula $m_{r}=$ $\frac{y_{1}-y_{0}}{x_{1}-x_{0}}$. Fazendo as escolhas como na situação anterior, teríamos $m_{r}=-2$. Como $s$ e $r$ são perpendiculares, vale a relação $m_{r} \cdot m_{s}=-1$, em que $m_{s}$ é o coeficiente angular da reta $S$. Devemos ter $m_{S}=1 / 2$. Conhecendo o coeficiente angular de $S$ e um ponto que pertence a ela, no caso a origem $0(0,0)$, basta substituir estes dados na fórmula $y-y_{0}=$ $m_{s}\left(x-x_{0}\right)$ para obtermos a equação da reta procurada. Nesse caso, temos $y-0=$ $\frac{1}{2}(x-0)$, o que nos dá $y=x / 2$ ou $x-2 y=0$.

Resolução 2 - técnica 2 (com uso de vetores). Como a reta $S$ é perpendicular à reta que contém $P$ e $Q$, ela é perpendicular ao vetor $P Q=(1-2,5-3)=(-1,2)$. A equação geral da reta é $A x+B y+C=0$, em que $(A, B)$ são coordenadas de um vetor perpendicular a reta. Podemos considerar, neste caso, $A=-1$ e $B=2$. Isso nos daria $-x+2 y+C=0$. Considerando que $O(0,0)$ pertence à reta, temos $-0+2(0)+C=$ 
0 , o que nos dá $C=0$. Assim, $-x+2 y=0$ seria a equação geral da reta s e $y=x / 2$ seria a equação reduzida.

Ademais, como consequências didáticas da experimentação das duas últimas tarefas, pode-se dizer que a resolução com vetores parece ser menos econômica do ponto de vista cognitivo, porém ao compreender essa resolução, o estudante se apropriaria ainda mais da noção de paralelismo e perpendicularismo entre vetor e reta, bem como do resultado fundamental da geometria analítica, que é "um ponto pertence a um objeto geométrico quando suas coordenadas satisfazem a equação deste objeto". Este resultado fundamental é a base da construção dos objetos geométricos via equação e é muito utilizado na GA, apesar de ser explicitamente pouco difundido. Além disso, na resolução com vetores, o estudante basicamente trabalharia com vetores e as expressões das retas, necessitando compreender apenas os significados de alguns coeficientes, enquanto que, na resolução sem vetores, seria necessário o uso de fórmulas adicionais, mesmo que simples.

No entanto, o trabalho com vetores no ensino de Matemática na Educação Básica não tem um amparo explícito na Base Nacional Comum Curricular (BRASIL, 2018), o que é um fator de restrição do uso do modelo de forma ampla no Ensino Médio. Por outro lado, essa restrição não impede que o modelo seja colocado em prática naquele nível de ensino, sendo necessário, para tanto, o conhecimento por parte dos docentes do referido modelo, o que pode ser feito pelas vias da formação docente inicial e continuada.

Ademais, acreditamos que as quatro tarefas apresentadas nesta discussão mostram que o uso de vetores não impacta numa elevação do nível de dificuldade nas resoluções das tarefas, ao invés disso, proporcionaria um ganho na transição entre GA do Ensino Médio e do Ensino Superior, visto que todas elas possibilitam ao estudante conhecer o objeto do conhecimento vetor e sua relação com retas e com os conceitos de paralelismo e perpendicularismo. Ressaltamos, ainda, que no componente curricular Geometria Analítica no Ensino Superior, ocorre um aprofundamento da teoria vetorial bem como o seu uso no espaço $\mathrm{R}^{3}$ e, uma vez ocorrido o estudo de vetores no Ensino Médio, tanto esse aprofundamento quanto sua versão no espaço poderia implicar em praxeologias mais consistentes e, por consequência, estudantes verdadeiramente em atividade matemática.

Após a explicitação do tipo de tarefa que o pretenso modelo didático-praxeológico comporta, apresentamos na Figura 10, a seguir, o desenho do seu percurso. Observe-se 
que nele o objeto vetor é destacado como ideia matemática articuladora de dois níveis de ensino. Assim, o elemento de transição, proposto no referido MDP, é a inclusão de vetor no Ensino Médio, o que torna o MDP um modelo alternativo a determinados modelos pautados, exclusivamente, nas orientações da BNCC.

Figura 10. Esquema representativo do MDP

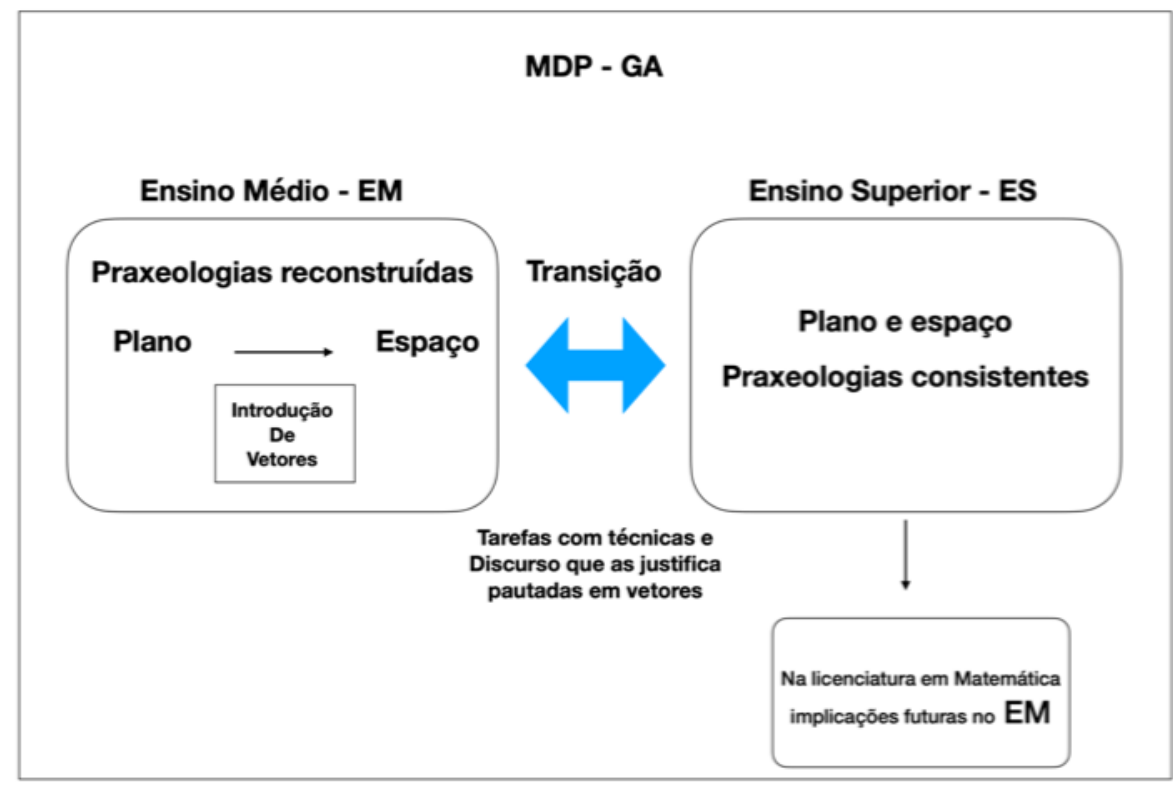

Fonte: Os autores.

Além do que foi explanado sobre a Figura 10, no Ensino Médio, na ideia de integrar vetores aos objetos do conhecimento que devem ser estudados, tem-se forte a noção de reconstrução de praxeologias matemáticas, que passam de exclusivamente construídas no plano para plano e espaço. Enquanto isso, inferimos que no Ensino Superior, na formação docente, por exemplo, as praxeologias matemáticas tornam-se mais consistentes e as implicações disso poderão ser notadas no Ensino Médio, a partir da atuação dos professores que tiveram uma vivência pautada no MDP na sua formação. Os elementos fundamentais desse modelo, que atuarão na transição entre níveis, são as tarefas, abordando a noção de vetores, tanto no que se refere às técnicas quanto em relação ao discurso que justifique tais técnicas.

\section{Considerações finais}

Primeiramente, faz-se necessário destacar o caráter provisional do MDP apresentado brevemente. Ele expressa a crença dos pesquisadores e não possui alicerce, 


\section{ENSIN@UFMS 2021}

ISSN 2525-7056

do ponto de vista institucional, nos documentos de orientação curricular, ao menos não de forma explícita. Entretanto, isso não impede que ele seja experimentado como uma criação do professor no seu fazer didático.

Quanto os resultados da investigação, destacamos as principais consequências didáticas do uso do MDP, a saber: economia nas resoluções das tarefas, que chamamos de economia cognitiva, e aproximação das praxeologias matemáticas de duas instituições complementares, a GA no Ensino Médio e a GA no Ensino Superior, o que é feito a partir do uso de vetores como noção articuladora. Em outras palavras, o uso de vetores é o elemento que possibilitaria uma transição harmoniosa, em tese, entre os dois níveis de escolaridade, e esse uso não apresenta, na prática, grandes complicações desde que sejam introduzido no ensino aspectos conceituais.

No que tange às restrições da investigação em que apresentamos alguns aspectos, elencamos: do ponto de vista prático, a ausência de referência explicitada na Base Nacional Comum Curricular (BRASIL, 2018) pode impedir que este modelo seja implementado em alguns contextos; da pesquisa propriamente dita, o fato de não ocorrer a experimentação do MDP proposto e apresentado aqui, nos impede de argumentar sobre seu alcance e mesmo sobre sua real viabilidade nas instituições de Ensino Médio.

E, para finalizar, sem esgotar a discussão, existem nuances do MDP que precisam ser estudadas, tais como, a possibilidade ou não de cobrir um currículo ou parte dele com tal modelo, a sua aplicabilidade com outras noções matemáticas como estruturantes e as possíveis criações didáticas que os docentes podem integrar ao MDP em suas práticas. De qualquer modo, tais aspectos apontam para a continuidade da investigação, como um mapeamento do alcance do modelo, tendo como noção articuladora vetores, mas com base nos dados empíricos.

\section{Referências}

ARTIGUE, M. Ingénierie Didactique. Recherches em Didactique des Mathématiques, Grenoble, v. 9, n. 3, p. 281-308, 1988.

BRASIL. Ministério da Educação. Base Nacional Comum Curricular. Brasília, 2018

CHEVALLARD, Y. L'analyse des pratiques enseignantes en Théorie Anthropologique du Didactique. Recherches en Didactique des Mathématiques, [s. I.], v. 19, n. 2, p. 221266, 1999. 
CHROBAK, R. Mapas conceituales y modelos didacticos de professors de química. In: CONFERENCE ON CONCEPT MAPPING, 2, CONGRESO INTERNACIONAL SOBRE MAPAS CONCEPTUALES, 2, 2006, San José, Costa Rica. Anais eletrônicos. San José: CMC, 2006, Sept. 5-8, 2006. Disponível em:

http://cmc.ihmc.us/cmc2006Papers/cmc2006-p215.pdf. Acesso em: 09 fev. 2010.

GRYMUZA, A. M. G.; RÊGO, R. G. A Teoria da Atividade: uma possibilidade no Ensino de Matemática. Revista Temas em Educação, [s. I.], v. 23, n. 2, p. 117-138, 2014.

Disponível em: https://periodicos.ufpb.br/index.php/rteo/article/view/20864. Acesso em: 05 mar. 2021.

GARCíA PÉREZ, F. F. Los modelos didácticos como instrumento de análisis y de intervención en la realidad educativa. Revista Electrónica de la Universidad de Barcelona, Barcelona, n. 207, 2000. Disponível em: http://www.ub.es/geocrit/b3w207.htm. Acesso em: 20 fev. 2021.

GARNICA, A. V. M. História Oral e educação Matemática. In: BORBA, M. C.; ARAÚJO, J. L. (Org.) Pesquisa Qualitativa em Educação Matemática. Belo Horizonte: Autêntica, 2004.

GRENDENE, M. V. C.; MELO, W. V. Metacognição e envelhecimento sob a luz do pensamento sistêmico: uma proposta de intervenção clinica. Revista Brasileira de Terapias Cognitivas, [s. I.], v. 4, n. 2, pp. 121-138, 2008.

GIL-PÉREZ, D.; CARVALHO, A. M. P. Formação de professores de Ciencias: tendências e inovações. São Paulo: Editora Cortez, 1995.

HENRIQUES, A.; ATTIE, J. P.; FARIAS, L. M. S. Referências teóricas da didática francesa: análise didática visando o estudo de integrais múltiplas com auxílio do software Maple. Educação Matemática Pesquisa: Revista do Programa de Estudos PósGraduados em Educação Matemática - PUC, São Paulo, v. 9, n. 1, pp. 51-81, 2007. Disponível em: https://revistas.pucsp.br/index.php/emp/article/view/585. Acesso em: 19. mar. 2021.

LUCAS, C.; FONSECA, C.; GÁSCON, J.; CASAS, J. O fenômeno didático institucional da rigidez e atomização das organizações matemáticas escolares. Bolema, Rio Claro (SP), v. 28 , n. 50, p. 1327-1347, dez. 2014.

MATHERON, Y. Analyser les praxéologies quelques exemples d'organisations mathématiques. Petit x, [s. I.], n. 54, p. 51-78, 2000.

MENEZES, P. J. D. Modelo didático-praxeológico alternativo para o ensino de objetos da Geometria Analítica: Elo entre o ensino médio e o superior. 2021. Dissertação (Metrado Profissional em Matemática em Rede Nacional- PROFMAT) Centro das Ciências Exatas e das Tecnologias, Universidade Federal do Oeste da Bahia. Barreiras, 2021. 
PORLÁN, R.; A. RIVERO Y.; MARTÍN, R. Conocimiento profesional y epistemología de los profesores I: teoría, métodos e instrumentos. Enseñanza de las Ciencias, [s. I.], v. 15, n. 2, pp. 155-171, 1997.

STEINBRUCH, A.; WINTERLE, P. Geometria Analítica. 2a edição, São Paulo: Makron Books, 1987.

\section{NOTAS}

\section{IDENTIFICAÇÃO DE AUTORIA}

Pedro José Defensor Menezes. Mestre em Matemática pela Universidade Federal do Oeste da Bahia.

Professor da Escola Estadual El Shadai, Barreiras, Ba, Brasil.

E-mail: pedrojdm@gmail.com

(D) https://orcid.org/0000-0002-3701-8728

Edmo Fernandes Carvalho. Doutor em Ensino, Filosofia e História das Ciências. Professor Adjunto. Membro do Programa de Mestrado Profissional em Matemática em Rede Nacional (Profmat). Universidade Federal do Oeste da Bahia, Centro das Ciências Exatas e das Tecnologias, Barreiras, Ba, Brasil.

E-mail: edmo.carvalho@ufob.edu.br

(1) http://orcid.org/0000-0002-6959-2652

Lauriclecio Figueredo Lopes. Mestre em Matemática. Professor Adjunto. Coordenador do Curso de Licenciatura em Matemática. Universidade Federal do Oeste da Bahia, Centro das Ciências Exatas e das Tecnologias, Barreiras, Ba, Brasil.

E-mail: lauriclecio@ufob.edu.br

(iD) https://orcid.org/0000-0002-3356-5644

\section{AGRADECIMENTOS}

Não se aplica.

\section{FINANCIAMENTO}

Não se aplica.

\section{CONSENTIMENTO DE USO DE IMAGEM}

Não se aplica.

\section{APROVAÇÃO DE COMITÊ DE ÉTICA EM PESQUISA}

Não se aplica.

\section{LICENÇA DE USO}

Autores mantêm os direitos autorais e concedem à revista ENSIN@ UFMS - ISSN 2525-7056 o direito de primeira publicação, com o trabalho simultaneamente licenciado sob a Licença Creative Commons Attribution (CC BY-NC-SA 4.0), que permite compartilhar e adaptar o trabalho, para fins não comerciais, reconhecendo a autoria do texto e publicação inicial neste periódico, desde que adotem a mesma licença, compartilhar igual.

\section{EDITORES}

Patricia Helena Mirandola Garcia, Eugenia Brunilda Opazo Uribe, Gerson dos Santos Farias.

\section{HISTÓRICO}

Recebido em: 14/09/2021 - Aprovado em: 25/11/2021 - Publicado em: 15/12/2021.

\section{COMO CITAR}


MENEZES, P. J. D; CARVALHO, E. F; LOPES, L. F. Modelo Didático-Praxeológico para Ensino de Vetores no Ensino Médio: Possibilidades de Trabalhos na Transição para o Ensino Superior. Revista ENSIN@ UFMS, Três Lagoas, v. 2, número especial, p. 164-185. 2021. 